\title{
Técnicas empleadas en la evaluación de la calidad de la caballa (Scomber japonicus peruanus), durante el proceso de descomposición: Una Revisión
}

\section{Techniques used in the evaluation of the quality of mackerel (Scomber japonicus peruanus) during the decomposition process: A Review}

\author{
Mónica del Rosario Saavedra ${ }^{1, *}$
}

\author{
${ }^{1}$ Bach., Universidad Nacional de Frontera - Sullana, Sullana, Perú, \\ \2015103067@unf.edu.pe, (D) https://orcid.org/0000-0002-9254-4202 \\ * Autor de Correspondencia: Tel. +51 902255265 \\ http://dx.doi.org/10.25127/riagrop.20213.701
}

http://revistas.untrm.edu.pe/index.php/RIAGROP revista.riagrop@untrm.edu.pe

Recepción: 30 de marzo 2021

Aprobación: 02 de junio 2021

Este trabajo tiene licencia de Creative Commons. Attribution-NonCommercial-ShareAlike $\quad 4.0$ International Public License - CC-BY-NC-SA 4.0

\section{Resumen}

En las últimas décadas, se realizaron diversas técnicas para la medición de la calidad de la caballa (Scomber japonicus peruanus). La selección de información sobre estas técnicas es un tema de investigación muy importante y esencial para realizar la clasificación de la frescura, estimar los cambios de calidad durante el proceso de descomposición de la caballa. Esta revisión presenta un análisis sistemático de la literatura referida a las técnicas empleadas en la medición de la calidad de la caballa. Además, se realizó un estudio de los principales cambios físicos, químicos, microbiológicos y sensoriales, así como, las técnicas y los principales problemas en la evaluación de calidad de esta especie. Se obtuvo información de las principales bases de datos (ScienceDirect, Springer, Wiley, Taylor and Francis, ACS Publications, Google Académico) que abarcaron los años 2007 hasta 2020, se obtuvieron un total de 88 artículos y se seleccionaron 43 estudios primarios. Los resultados obtenidos determinaron que la mayoría de los estudios primarios utilizaron las técnicas no tradicionales como: HPLC, cromatografía de gases, colorimetría, PCR-RFLP, etc. Estas técnicas presentaron diversos problemas en su aplicación. Asimismo, se identificaron técnicas no 
tradicionales como: espectroscopía, microscopía, biosensores, etc. que demostraron ser eficientes en la evaluación de la calidad de la caballa.

Palabras clave: Descomposición, evaluación de la calidad, técnicas, caballa, Scomber japonicus.

\begin{abstract}
In the last decades, several techniques have been used to measure the quality of mackerel (Scomber japonicus peruanus). The selection of information on these techniques is a very important and essential research topic for freshness grading, estimating quality changes during the mackerel decomposition process, etc. This review presents a systematic analysis of the literature on the techniques used in the measurement of mackerel quality. In addition, a study is made of the main physical, chemical, microbiological and sensory changes, as well as the techniques and the main problems in the quality evaluation of this species. Information was obtained from the main databases (ScienceDirect, Springer, Wiley, Taylor and Francis, ACS Publications, Google Scholar) covering the years 2007 to 2020, obtaining a total of 88 articles and selecting 43 primary studies. The results obtained determined that the majority of the primary studies used non-traditional techniques such as: HPLC, gas chromatography, colorimetry, PCR-RFLP, etc. These techniques presented various problems in their application. Likewise, non-traditional techniques such as: spectroscopy, microscopy, biosensors, etc. were identified. These techniques proved to be efficient in the evaluation of mackerel quality.
\end{abstract}

Keywords: Decomposition, quality evaluation, techniques, mackerel, Scomber japonicus.

\section{INTRODUCCIÓN}

La caballa es una de las especies pelágicas más capturadas y comercializadas, además, se ubica entre los veinticinco primeros puestos en el mundo. Los países de mayor producción y exportación a nivel mundial son China, Japón, Noruega, Reino Unido, Islandia y Corea (Ministerio de Agricultura, 2017). En el Perú, la caballa es considerado un recurso de gran demanda debido a sus propiedades nutricionales y se encuentra en el tercer puesto de importancia pesquera entre las especies pelágicas (Cruces et al., 2014).

Actualmente, existe un mayor desembarque para consumo humano directo de caballa (17.3 $\%$ ), que representa 28870 toneladas (IMARPE, 2019). Además, el volumen de desembarque se incrementó del año 2018 al 2020 en un $7.5 \%$ (PRODUCE, 2020). Los principales mercados peruanos de exportación de caballa congelada son Nigeria (12\%), Costa de Marfil (18\%) y Brasil (8 \%) (SIICEX, 2019).

Asimismo, el consumo de caballa es una fuente importante de proteínas $(19.5 \%)$, grasas saturadas (4.9\%) y colesterol (70.0\%). Además, otorga micronutrientes esenciales como vitaminas (A, B y D), minerales (calcio, yodo, zinc, hierro y selenio) y ácidos grasos poliinsaturados como el omega 3 y 6; de los cuales predomina el omega 3 (FAO, 2014).

Estas características de buena calidad nutricional de la caballa influyen en su proceso de deterioro, principalmente, en los métodos de almacenamiento y sus parámetros de tiempo y temperatura (Jun-Hu Cheng et al., 2015).

Debido a su rápida descomposición, puede generar compuestos volátiles si no se lleva un buen control de la cadena de frío, que da lugar 
a sabores y olores desagradables asociados con el deterioro, producto de las bacterias Gramnegativas (Psychrobactersp sp., P. immobilis, Shewanella sp., Vibrio sp., P. cibarius, etc.) (de Alba et al., 2012).

La caballa se deteriora debido a la oxidación de sus ácidos grasos como omega-3: ácido docosahexaenoico (DHA) y ácido eicosopantaenoico (EPA). Estos originan la rancidez y pérdida de calidad (Cropotova et al., 2019). Después de la muerte del pez, se produce una rápida disminución del $\mathrm{pH}$, que provocan alteraciones en la integridad muscular en la caballa (Sone et al., 2019). Yu et al. (2020) mencionan que el inicio de la pérdida de la calidad de la caballa, se genera durante el almacenamiento post mortem, que ocasiona la decoloración, degradación de proteínas (sarcoplásmicas y miofibrilares), pérdida por goteo, descomposición de nucleótidos $\mathrm{y}$ acumulación de compuestos nitrogenados. Además, se le atribuyen los cambios físicos como la textura y el color.

La calidad de la carne de caballa se analiza tradicionalmente de la siguiente manera:

- Métodos sensoriales. Estos se basan en cambios de apariencia, olor, color, sabor y textura, es decir, método del índice de calidad (Simeonidou et al. 1997; Alberio et al., 2014; Jun-Hu Cheng et al., 2014; Aubourg et al. 2013).

- Métodos de detección química. Estos métodos se basan en técnicas tradicionales como: cromatografía líquida de alta resolución (HPLC) (He et al., 2020; Sveinsdóttir et al., 2020; Bae y Lim, 2012; Sarkardei y Howell 2007).

- Cromatografía de gases (Vázquez et al. 2013; Chow et al., 2011).
- Métodos microbianos, basados en la técnica de recuentos viables totales (Otero et al. 2019; Ezquerra et al. 2016; Senturk y Alpas, 2012; Stamatis y Arkoudelos 2007).

- Fragmentos de restricción de longitud polimórfica, conocida como PCR-RFLP (Bak et al. 2014; Jun-Hu Cheng et al., 2015).

Estas técnicas presentan ciertas desventajas tales como pérdidas de las muestras, son tediosas, requieren de mucho tiempo, son destructivas, ofrecen información limitada, son costosas, entre otras. Debido a esto, diversas investigaciones desarrollan nuevas técnicas no tradicionales para la evaluación de la calidad de la caballa como:

- Resonancia Magnética Nuclear (RMN) (Gudjónsdóttir et al., 2019; Albertos et al. 2017).

- Microscopía de fluorescencia y electrónica de barrido (Cropotova et al. 2019; Hashimoto et al., 2016).

- Espectroscopía de masas (MS/MS), infrarrojo medio por transformada de Fourier (MIRFTIR), ultravioleta visible, NIR, etc. (Fidalgo et al. 2014; Lv et al. 2019; Pazos et al. 2015; ElMasry y Wold, 2008).

- Técnicas de visión por computadora (Mozuraityte et al., 2020; Kunjulakshmi et al., 2020).

- Técnicas de biosensores como el Sistema de inspección óptica (OIS) (Choi et al., 2020; Chow et al., 2011; Moriya et al. 2019).

En consecuencia, el objetivo de esta revisión fue dar a conocer el avance del uso de las técnicas empleadas en la evaluación de la calidad de la caballa (Scomber japonicus peruanus) durante su proceso de descomposición. Posteriormente a esta parte introductoria, la revisión se dividió 
por secciones: la sección 2 presenta una breve contextualización acerca de las técnicas de la evaluación de calidad del pescado y sus aplicaciones en la caballa; la sección 3 detalla el método de revisión sistemática desarrollado; la sección 4 describe el análisis de los resultados, y para finalizar la sección 5 precisa las conclusiones y trabajos futuros.

\section{MATERIALES Y MÉTODOS}

\subsection{Contextualización}

Las técnicas usadas en la evaluación de la calidad del pescado proceden de la década de los 70 y comienzo de los 80, en Tasmania. El primer método desarrollado fue el índice de la calidad, empleado para medir la frescura y la vida útil del pescado (Bernardi et al., 2013).

Además, se desarrollaron métodos microscópicos para determinar los cambios estructurales; y métodos químicos para establecer niveles de tolerancia a través de indicadores de deterioro, que usaron técnicas como la colorimetría, destilación, extracción, potenciometría, etc. (FAO, 1998).

La industria alimentaria ha empleado diversas técnicas para la evaluación sensorial, física, química y microbiológica de la calidad de la caballa. Por esta razón, existen varias maneras de clasificarlas; por ejemplo, técnicas tradicionales como: cromatografía líquida de alta resolución (HPLC), cromatografía de gases, colorimetría, potenciometría, recuentos viables totales, PCR-RFLP, índice de la calidad, etc.

Por otro lado, se encuentran las técnicas no tradicionales como: Resonancia Magnética Nuclear (RMN), espectroscopía de infrarrojo medio por transformada de Fourier (MIRFTIR), la microscopía de fluorescencia, biosensores, etc. Estas técnicas son las nuevas tecnologías que vienen siendo empleadas en la evaluación de la calidad de la caballa.

Independientemente, ambos tipos de técnicas se diferencian entre sí debido a la complejidad de su ejecución, la capacidad de respuesta rápida, entre otros. Para saber sobre las ventajas que ofrecen estas técnicas le recomendamos al lector revisar los trabajos de Cheng et al. (2013) y Wu et al. (2019).

Las técnicas tradicionales anteriormente mencionadas desempeñan un papel importante en la evaluación e inspección de la calidad y la inocuidad de la caballa porque algunas de ellas se han utilizado como estándares de calidad por su relativa validez y precisión.

Por otra parte, las técnicas no tradicionales han despertado un gran interés debido a la alta especificidad, conveniencia, no destructivo, no invasivo, respuesta rápida y eficacia, que demuestran un gran potencial para la detección de patógenos, contaminación, cambios en la estructura de las proteínas, la oxidación de lípidos, y para el seguimiento del deterioro en la caballa. Todo esto con la finalidad de confirmar si es segura para el consumo y el comercio internacional (Cheng et al. 2013).

Con relación a lo antes mencionado, en la presente revisión sistemática, se pretendió demostrar el avance en el uso de las técnicas en la evaluación de la calidad de la caballa.

\subsection{Revisión de la literatura}

De acuerdo con Ponce-Corona et al. (2020), en esta sección se explicó el procedimiento que siguieron para elaborar la revisión sistemática de la literatura y utilizaron el método basado en las sugerencias detalladas en Kitchenham 
(2007), que se emplearon últimamente por Miramontes et al. (2016), Linares-Espinós et al. (2018), Muñoz et al. (2019), entre otros. Este método está compuesto por tres etapas principales: planificación de la revisión, desarrollo de la revisión y reporte de resultados.

\subsection{Planificación de la revisión}

La primera etapa de la revisión sistemática es la planificación. Esta etapa realiza el protocolo que dirigirá la revisión. En esta revisión se tomaron en cuenta las siguientes actividades: Identificación de la necesidad de revisión, formulación de preguntas de investigación, creación de la cadena de búsqueda $\mathrm{y}$ identificación de las fuentes de datos.

\subsubsection{Identificación de la necesidad para realizar la revisión sistemática}

Cuando se sabe que caballa es un alimento altamente perecedero, la industria de procesamiento de pescado, en su preocupación por atender a las necesidades de un mercado cada vez más exigente, busca mejorar las metodologías de control de pérdida de calidad de la caballa. Por esta razón, es de interés elaborar una revisión de la literatura, para determinar qué técnicas vienen siendo desarrolladas en la evaluación de la calidad de la caballa (Scomber japonicus peruanus), que permite identificar los cambios físicos, químicos, microbiológicos y sensoriales dados en los procesos de descomposición.

\subsubsection{Formulación de las preguntas de investigación}

Se formularon las siguientes preguntas de investigación.

- PI01: ¿Cuáles son las técnicas empleadas para la evaluación de la calidad de la caballa durante el proceso de descomposición?
- PI02: ¿Cuáles son las principales fuentes de información en base a artículos científicos en el tema de la evaluación de la calidad de la caballa durante el proceso de descomposición?

- PI03: ¿Cuáles son los principales problemas en la aplicación de las técnicas utilizadas para la determinación de la calidad de la caballa durante el proceso de descomposición?

- PI04: ¿Qué cambios físicos, químicos, microbiológicos y sensoriales han sido los más analizados por las técnicas de evaluación de la calidad de la caballa durante el proceso de descomposición?

- PI05: ¿Qué técnicas han sido las más aplicadas para la evaluación de la calidad de la caballa durante su proceso de descomposición?

- PI06: ¿Cuáles son las mejores técnicas para aplicar en la determinación de la calidad de la caballa durante su proceso de descomposición?

\subsubsection{Creación de la cadena de búsqueda}

En esta fase, se identificaron las palabras claves, que fueron obtenidas de las preguntas de investigación.

Los principales problemas detectados en la evaluación de la calidad de la caballa fueron: descomposición, evaluación de la calidad y técnicas. Se optó por dividir la especie de la caballa por el tipo de especie, dando como resultado las palabras claves: Decomposition, Quality evaluation, Techniques, Mackerel, Scomber scombrus, Scomber japonicus.

La elaboración de la cadena de búsqueda se logró a partir de la unión de las palabras clave y el uso de los operadores lógicos AND y OR. En 
efecto, se obtuvo la siguiente cadena: [Quality or Decomposition or Quality evaluation or techniques] AND [mackerel or Scomber japonicus].

Para este estudio, se entiende por evaluación de la calidad a la forma, o aspecto estético, y frescura, o el nivel de deterioro que ha sufrido la caballa. Se puede estimar mediante parámetros físicos, químicos, microbiológicos y sensoriales mediante la comprobación la presencia o ausencia de propiedades (composición, textura, pureza, estado, color, aroma, etc.) (FAO, 1998).

La descomposición se refiere a las alteraciones complejas post mortem, que experimenta la caballa por la acción de sus propias enzimas, bacterias y reacciones químicas. Esto genera pérdidas en las propiedades originales; la apariencia y el sabor son desagradables; generando enfermedades o intoxicaciones. Esta degradación la vuelve no apta para el consumo humano y sin valor comercial.

\subsubsection{Identificación de las fuentes de datos}

De acuerdo con Ponce-Corona et al. (2020) y con las sugerencias planteadas por Kitchenham (2007) acerca de las lecciones estudiadas para los procedimientos de búsqueda de datos, se optó por usar las siguientes fuentes de datos: ScienceDirect, Springer, Wiley, Taylor and Francis, ACS Publications y Google Académico.

\subsection{Desarrollo de la revisión}

La revisión sistemática tiene como objetivo identificar la mayor cantidad de estudios primarios que sean posibles, si se aplica un plan de búsqueda neutral. En efecto, las preguntas de investigación se resolverán a través de los estudios primarios. La revisión sistemática se llevará a cabo en dos actividades: la selección los estudios primarios y el desglose $y$ simplificación de la información (Kitchenham, 2007).

\subsubsection{Seleccionar los estudios primarios}

Para demostrar que los estudios primarios se han obtenido de una manera imparcial en la búsqueda, se precisarán algunos criterios de inclusión y exclusión. Asimismo, se realizará un método específico (Miramontes et al., 2016).

Criterios de inclusión: estudios con idioma inglés (CI01), estudios publicados durante el periodo de enero del 2007 y diciembre de 2020 (CI02), estudios que lleven en el título por lo menos dos palabras clave (CI03) y estudios vinculados con la calidad de la caballa (descomposición, evaluación de la calidad y técnicas) (CI04).

Criterios de exclusión: estudios duplicados (CE01), estudios que estén fuera del periodo comprendido (CE02) y estudios que no se relacionan con la evaluación de la calidad de la caballa (CE03).

Para la selección de los estudios primarios se realizó el siguiente procedimiento:

1. Aplicar la cadena de búsqueda a la fuente de datos establecida.

2. Usar los criterios de inclusión CI01 y CI02.

3. Usar el criterio de inclusión CI03.

4. Leer resumen, metodología y conclusiones; asimismo, emplear el criterio de inclusión CI04 y los criterios de exclusión (Muñoz et al., 2019).

En la tabla 1, se observa el número de trabajos de investigación publicados dentro del periodo establecido para el estudio de revisión (véase columna 2). De la misma manera, se puede 
visualizar la cantidad de estudios primarios seleccionados (véase columna 3). Cabe mencionar, que a lo largo del proceso de búsqueda se encontraron duplicidad de estudios. Por consiguiente, después de emplear el método determinado para la selección de los estudios primarios, se seleccionaron 43 estudios.

Tabla 1. Número de investigaciones publicadas y estudios primarios

\begin{tabular}{ccc}
\hline $\begin{array}{c}\text { Fuentes de } \\
\text { datos }\end{array}$ & $\begin{array}{c}\text { Resultados de la } \\
\text { cadena de búsqueda }\end{array}$ & $\begin{array}{c}\text { Estudios } \\
\text { primarios }\end{array}$ \\
\hline $\begin{array}{c}\text { ScienceDirect } \\
\text { Springer }\end{array}$ & 40 & 21 \\
Wiley & 12 & 10 \\
Taylor and \\
Francis \\
ACS
\end{tabular}

\subsubsection{Extracción y simplificación de la información}

Para llevar a cabo la extracción de la información, los estudios primarios se organizaron a través del modelo de matriz de consistencia y fue un método de investigación fundamental para realizar el estado del arte. En esta actividad se diseñó una hoja de cálculo en la que se seleccionaron los siguientes datos de cada estudio primario: autor, año, título, revista, especie, objetivo, técnicas empleadas, métodos, parámetros evaluados (físicos, químicos y microbiológicos), resultados y problemas.

\section{RESULTADOS Y DISCUSIÓN}

En este apartado se presentan los resultados adquiridos de la revisión sistemática de la literatura, los mismos que dan a conocer de manera general el panorama de las diferentes técnicas empleadas para la evaluación de la calidad de la caballa durante el proceso de descomposición. Los resultados se planifican en cada pregunta de investigación.

PI01: ¿Cuáles son las técnicas empleadas para la evaluación de la calidad de la caballa durante el proceso de descomposición?

Los trabajos de investigación seleccionados se analizaron desde el año 2007 hasta el 2020, para conocer la frecuencia y evolución de las técnicas evidenciadas en las publicaciones. La tabla 2 presenta, de manera detallada, los resultados de este proceso de análisis y se aprecia las diferentes técnicas empleadas para cada tipo de método de evaluación, que han sido relacionados en la medición de la calidad de la caballa durante su proceso de descomposición.

PI02: ¿Cuáles son las principales fuentes de información, en base a artículos científicos, sobre el tema de la evaluación de la calidad de la caballa durante el proceso de descomposición?

En la presente revisión sistemática, se han obtenido 43 estudios que forman parte del grupo de estudios primarios $y$ fueron publicados en diferentes revistas. En la figura 1, se observan las revistas más destacadas en el tema de evaluación de la calidad de la caballa emplearon diversas técnicas, tales como: las revistas Innovative Food Science and Emerging Technologies, LWT - Food Science and Technology, Food Chemestry, International Journal of Food Science and Technology, de la editorial Elsevier y Food Science and Technology, de la editorial Fisheries Science. En las revistas mencionadas anteriormente se han publicado 12, 4, 4, 4 y 4 de 
los estudios seleccionados en esta revisión

respectivamente.

Tabla 2. Técnicas empleadas para la evaluación de la calidad de la caballa durante el proceso de descomposición

\begin{tabular}{|c|c|c|}
\hline Método & Técnicas & Equipo/instrumento/reactivo \\
\hline Sensorial & Índice de la calidad & Pruebas discriminativas y descriptivas \\
\hline \multirow[t]{10}{*}{ Químico } & Cromatografía líquida de alta resolución & Equipo HPLC \\
\hline & Cromatografía de gases & Cromatógrafo de gases \\
\hline & Neutralización y destilación & Kjeldahl \\
\hline & titulación & \\
\hline & Cromatografía líquida LC - MS & Cromatógrafo y espectrofotómetro \\
\hline & Calorimetría de barrido diferencial & Medidor de flujo de calor \\
\hline & Índice de fluidez & Medidor de fluencia \\
\hline & Extracción & Soxhlet \\
\hline & Cromatografía de capa fina TLC & Cromatógrafo \\
\hline & ELISA & Kits de prueba rápida \\
\hline \multirow[t]{3}{*}{ Físico } & Análisis de textura & Texturómetro \\
\hline & Potenciometría & pH-metro \\
\hline & Colorimetría & Tiocianato férrico \\
\hline \multirow[t]{4}{*}{ Microbiológico } & Recuentos viables totales & Agar de hierro \\
\hline & Recuentos viables totales & Caseína-agar \\
\hline & Recuentos viables totales & Agar de levadura \\
\hline & PCR-RFLP & \\
\hline Biosensores & Inspección óptica automatizada & Cámaras móviles \\
\hline \multirow[t]{6}{*}{ Espectroscópico } & Resonancia Magnética Nuclear (RMN) & Equipo RMN \\
\hline & $\begin{array}{l}\text { Espectroscopía de infrarrojo medio por } \\
\text { transformada de Fourier (MIR-FTIR) }\end{array}$ & Espectrofotómetro \\
\hline & Espectrometría de masas (MS / MS) & Espectrofotómetro \\
\hline & Espectrofotómetro de fluorescencia & Espectrofotómetro \\
\hline & Espectroscopía ultravioleta-visible, & Espectrofotómetro \\
\hline & Espectroscopía NIR & Espectrofotómetro \\
\hline \multirow[t]{2}{*}{ Microscópico } & Microscopía de fluorescencia & Microscopio \\
\hline & Microscopía electrónica de barrido & Microscopio \\
\hline
\end{tabular}

PI03: ¿Cuáles son los principales problemas, en la aplicación de las técnicas utilizadas, para la determinación de la calidad de la caballa durante el proceso de descomposición?

En la figura 2, se muestra un gráfico circular que brinda la información concerniente a los principales problemas de las técnicas, que han sido empleadas en la evaluación de la calidad de la caballa. Se observa que la mayoría de las técnicas requieren de mucho tiempo y preparación de la muestra, son costosas y generan pérdida de las muestras. Además, se identificaron otros problemas en la presente revisión.

PI04: ¿Qué cambios físicos, químicos, microbiológicos y sensoriales han sido los más analizados por las técnicas de evaluación de la 
calidad de la caballa durante el proceso de descomposición?

En la figura 3, se observa los tipos de cambios que han sido evaluados en la calidad de la caballa entera. Cada estudio ha sido agrupado por el tipo de cambio (físico, químico, microbiológico y sensorial). Los estudios químicos, físicos, microbiológicos y sensoriales han representado 37, 23, 3 y 9 investigaciones, respectivamente.

En la figura 4, se aprecia los parámetros de calidad más analizados por las técnicas en la evaluación de la calidad de la caballa. Se determinó que la oxidación lipídica fue el parámetro más evaluado, que tuvo 21 publicaciones. Del mismo modo, 11 publicaciones evaluaron el $\mathrm{pH}$ y dentro de los parámetros físicos, 12 publicaciones evaluaron los cambios en la textura de la caballa.

PI05: ¿Qué técnicas han sido las más utilizadas en la evaluación de la calidad de la caballa durante su proceso de descomposición?

En la figura 5, se observa las técnicas más utilizadas en la evaluación de la calidad de la caballa, en 11 estudios emplearon la técnica de cromatografía de gases, 9 estudios realizaron el índice de la calidad, 8 estudios emplearon los Recuentos Viables Totales y 7 utilizaron la técnica de Cromatografía de alto rendimiento (HPLC).

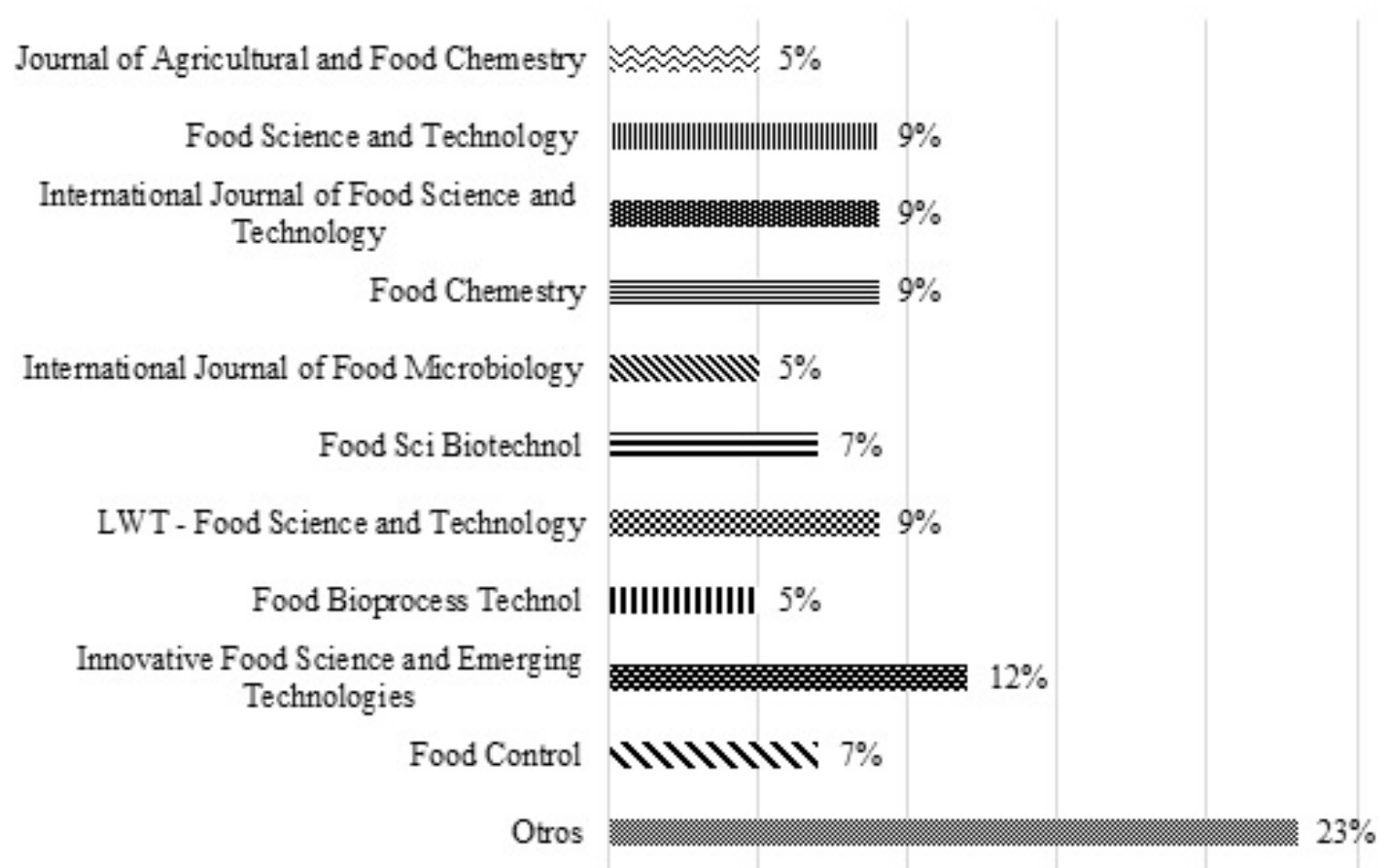

Figura 1. Evaluación de la calidad de la caballa en revistas científicas de base de datos. 


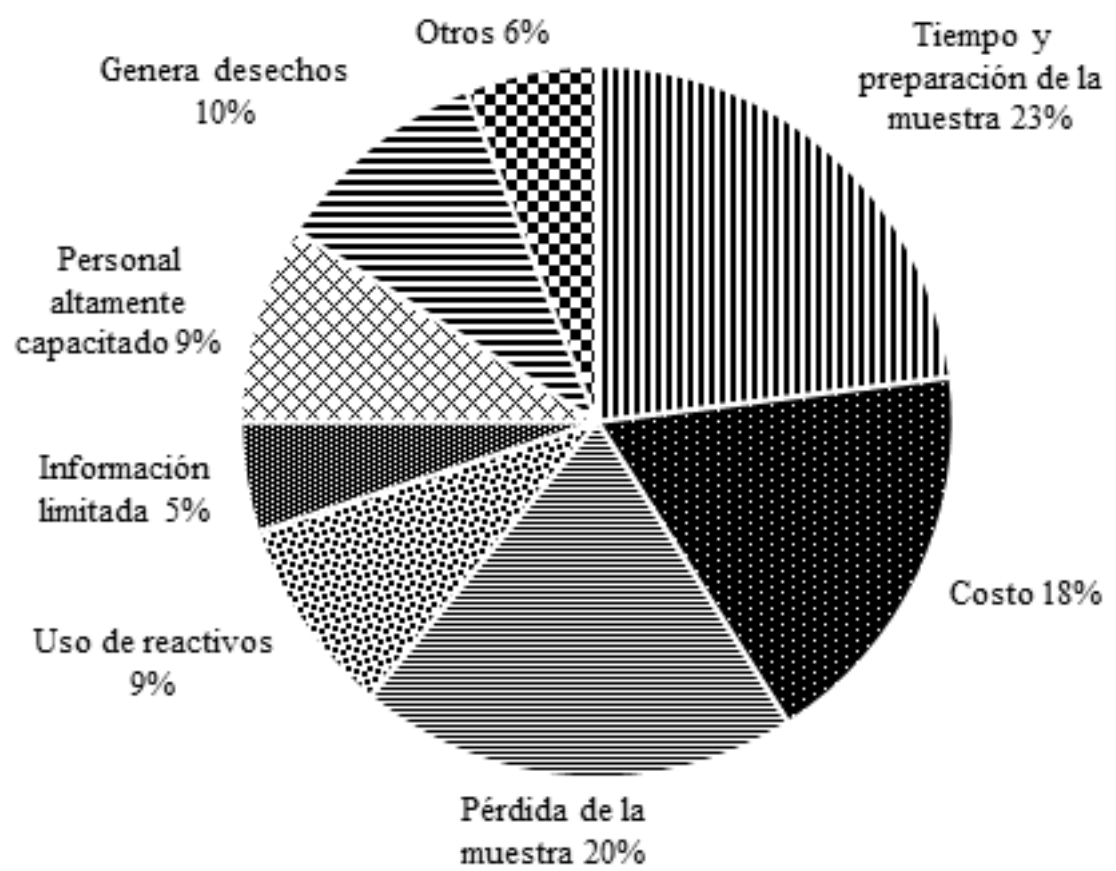

Figura 2. Principales problemas de las técnicas empleadas para la evaluación de la calidad de la caballa.

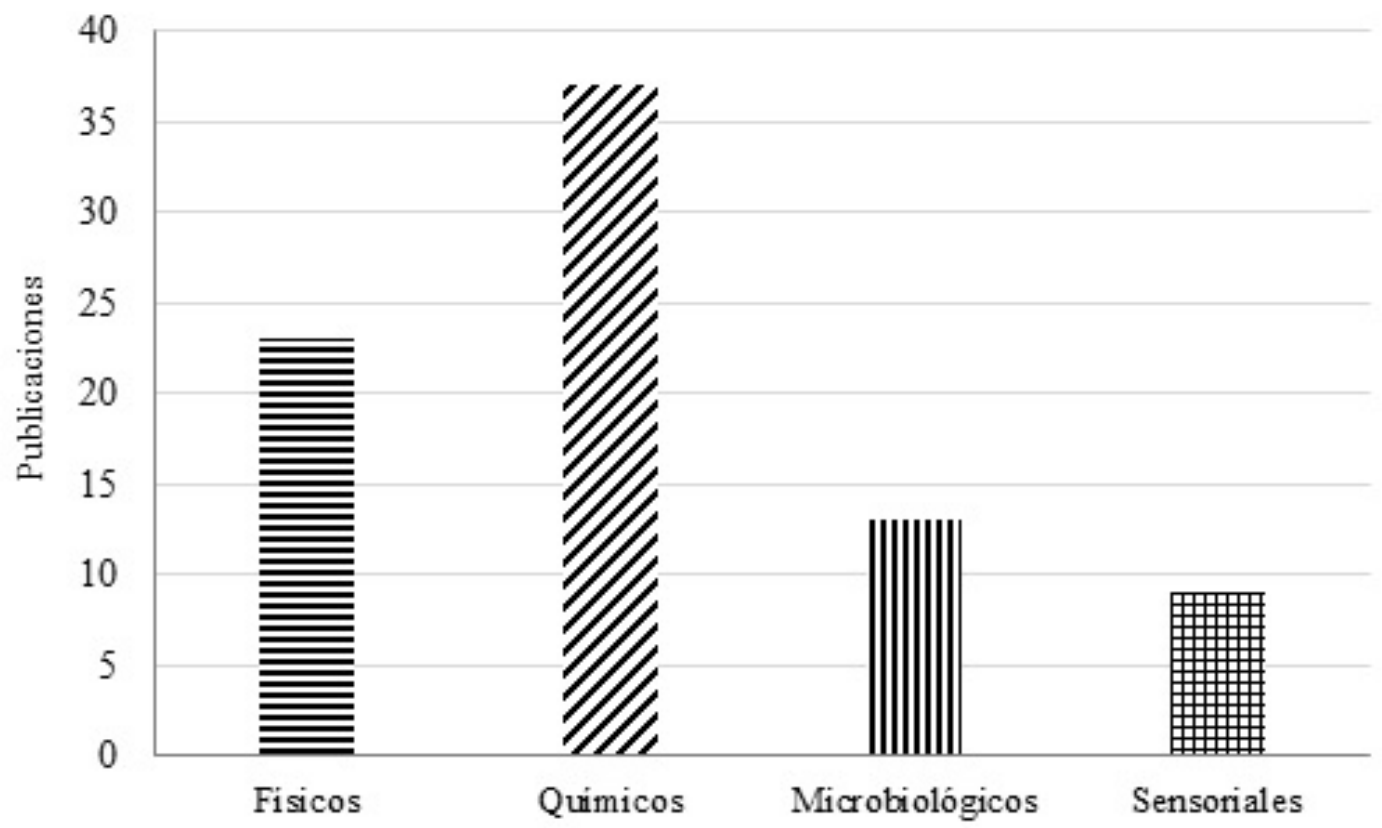

Figura 3. Cambios físicos, químicos, microbiológicos y sensoriales evaluados en la evaluación de la calidad de la caballa. 


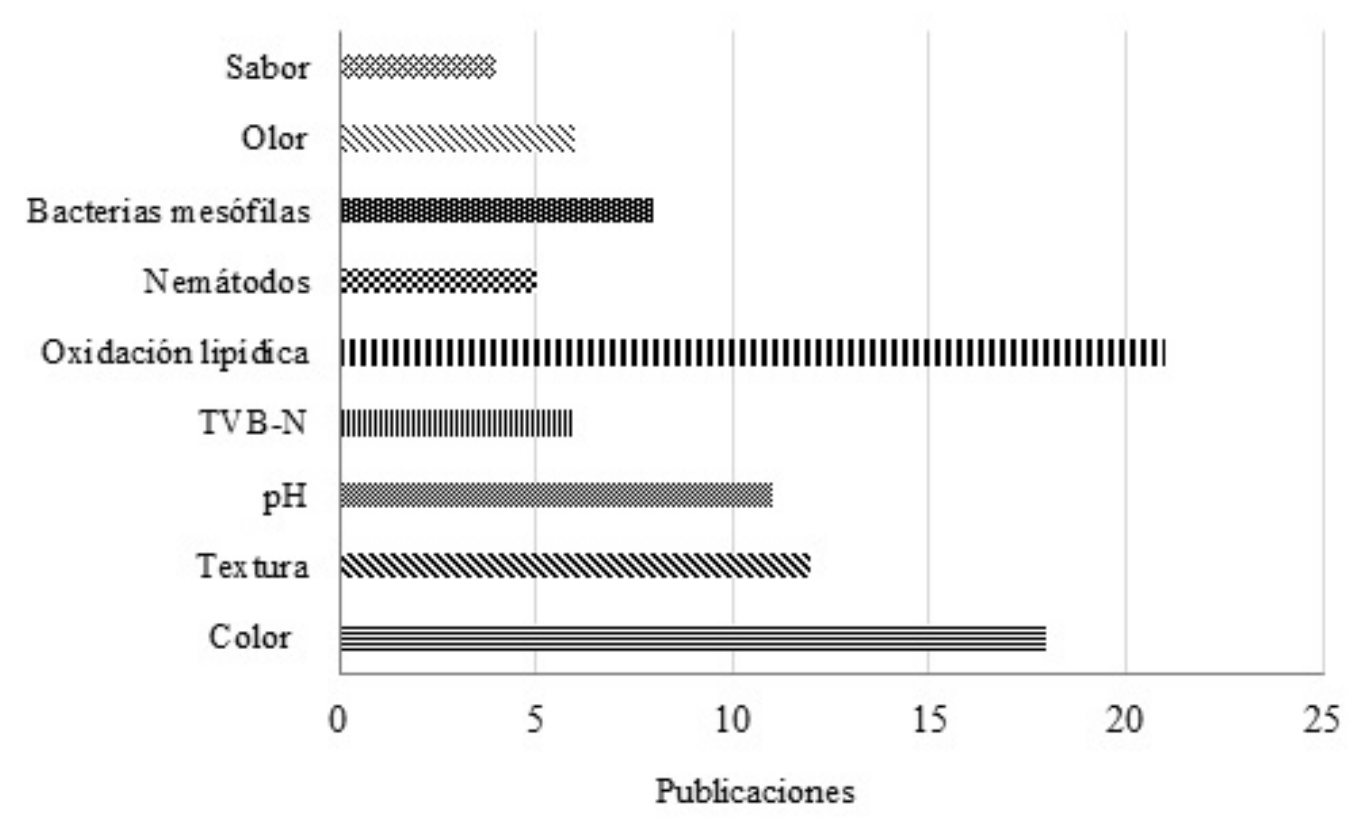

Figura 4. Parámetros físicos, químicos, microbiológicos y sensoriales más analizados por las técnicas de evaluación de la calidad de la caballa.

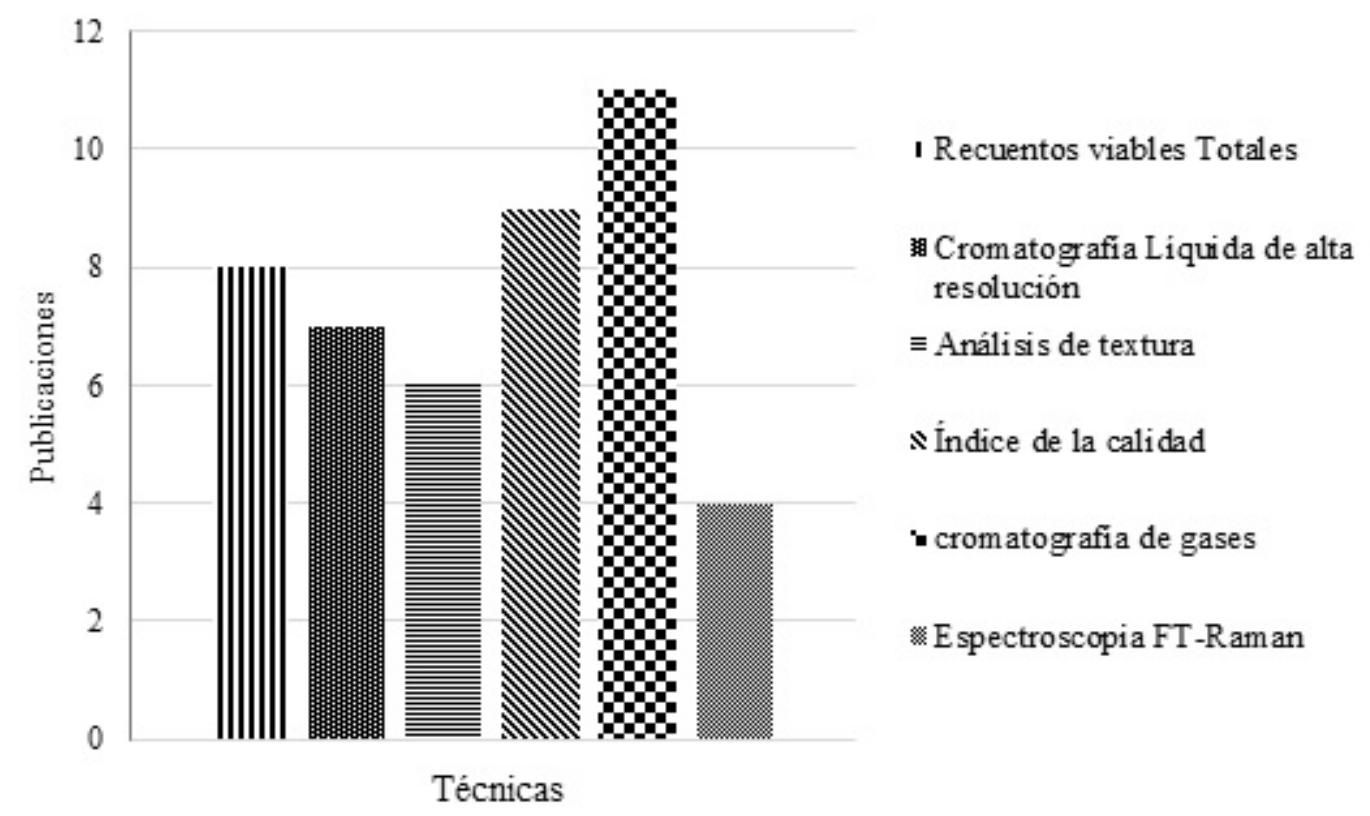

Figura 5. Técnicas más analizadas en la evaluación de la calidad de la caballa.

PI06: ¿Cuáles son las mejores técnicas para aplicar en la determinación de la calidad de la caballa durante su proceso de descomposición? Durante el procesamiento de datos, se pudo evidenciar que la mayoría de estudios emplearon las técnicas convencionales, tal y como se observa en la tabla 2. Las desventajas de estas técnicas son: requieren de mucho tiempo y preparación de la muestra, son costosas, generan pérdidas de las muestras, son destructivas, etc. (Bak et al. 2014); (Suh et al. 2017); (Choi et al., 2020); (Hashimoto $\mathrm{y}$ 
Yamashita 2019). Sin embargo, se identificaron nuevas tecnologías, como técnicas de biosensores, técnicas de espectroscopía y microscopía (Choi et al., 2020); (Hernandez et al. 2014); (Cropotova et al. 2019) cuyas ventajas son: la capacidad de detección rápida, no requieren de tiempo y preparación de las muestras, no son destructivas y se pueden emplear en línea de proceso. Estas son las mejores técnicas para aplicar en la determinación de la calidad de la caballa durante su proceso de descomposición.

\section{CONCLUSIONES}

En el presente trabajo de investigación, se realizó una revisión sistemática de la literatura referente a las técnicas empleadas en la medición de la calidad de la caballa. Por lo tanto, se lograron determinar los cambios físicos, químicos, microbiológicos y sensoriales que se presentaron durante el proceso de descomposición de la caballa.

En efecto, la evaluación de la calidad de la caballa ha sido ampliamente abordada durante las últimas décadas. Por consiguiente, se han aplicado diferentes técnicas. La mayoría de los estudios primarios se centraron en las técnicas no tradicionales como: la cromatografía de gases, índice de la calidad, cromatografía de alto rendimiento HPLC, recuento total de aeróbicos, etc. Se observó que los principales problemas de estas técnicas requieren de mucho tiempo y preparación de la muestra, son costosas, generan pérdidas de las muestras, son destructivas, presentan información limitada y requieren de personal altamente calificado.

Por otro lado, se han empleado técnicas no tradicionales, entre ellas: la espectroscopia FTRaman, infrarrojo medio por transformada de
Fourier (MIR-FTIR), espectrometría de masas (MS/MS), Sistema de inspección óptica (OIS), Microscopía de fluorescencia, etc.

Se analizaron diversos cambios en la calidad de la caballa, por ende, se determinó que la mayoría de los estudios primarios analizaron los cambios químicos. Por ende, los más evaluados fueron: la oxidación lipídica, $\mathrm{pH}$ y la determinación de Nitrógeno Básico Volátil Total. Asimismo, se determinaron otros cambios relevantes como: el color, la textura, el sabor, el olor, nemátodos y bacterias mesófilas.

Para finalizar, se concluye que de acuerdo al análisis de los estudios primarios en la presente revisión sistemática; se observó que las técnicas no tradicionales como: la espectroscopía, la microscopía y biosensores, obtuvieron un coeficiente de determinación ( $\mathrm{R} 2$ entre $0.79 \mathrm{y}$ 0.99). La espectrometría de masas (MS / MS) fue la de menor ajuste y la espectroscopía de infrarrojo medio por transformada de Fourier (MIR-FTIR), la de mayor ajuste. Estas técnicas demuestran ser excelentes alternativas para la evaluación de la calidad de la caballa durante su descomposición. Además, brindan información y detección rápida en línea de la calidad de la caballa, no son destructivas, ni generan desechos. Por lo tanto, contribuyen con el medio ambiente y generan mayores rendimentos económicos que evitan las pérdidas de las muestras.

De acuerdo con lo antes mencionado, se aconseja hacer un mayor uso de estas técnicas, también se requiere una mayor investigación en estas nuevas tecnologías. Es necesario examinarlas con más detalle para desarrollar y optimizar mejores sistemas de evaluación. Es decir, modelos multivariados de calibración y validación o análisis quimiométricos, para que 
puedan ser aplicados y controlen diversos parámetros fisicoquímicos y microbiológicos que permitan aumentar la precisión de la medición, confiabilidad y predicción de la calidad de la caballa.

\section{Agradecimientos}

La autora agradece a la Universidad Nacional de Frontera - Sullana, al asesor el Dr. Wilson, por su paciencia y enseñanzas. También agradezco a la Ing. Alba, por haber resuelto mis dudas cuando lo necesité.

\section{Referencias}

Alberio, G. R. A., Barbagallo, R. N., Todaro, A., Bono, G., \& Spagna, G. (2014). Effect of freezing/thawing process in different sizes of blue fish in the Mediterranean through lysosomal enzymatic tests. Food Chemistry, 148, 47-53.

Albertos, I., Martín-Diana, A. B., Cullen, P. J., Tiwari, B. K., Ojha, S. K., Bourke, P., \& Rico, D. (2017). Effects of dielectric barrier discharge (DBD) generated plasma on microbial reduction and quality parameters of fresh mackerel (Scomber scombrus) fillets. Innovative Food Science \& Emerging Technologies: IFSET: The Official Scientific Journal of the European Federation of Food Science and Technology, 44, 117-122.

Bae, J. H., \& Lim, S. Y. (2012). Chemical composition, antiproliferative and antioxidant properties of lipid classes in ordinary and dark muscles from chub mackerel (Scomber japonicus). Food and Chemical Toxicology: An International Journal Published for the British Industrial Biological Research Association, 50(34), 823-828.

Bak, T.-J., Jeon, C.-H., \& Kim, J.-H. (2014). Occurrence of anisakid nematode larvae in chub mackerel (Scomber japonicus) caught off Korea. International Journal of Food Microbiology, 191, 149-156.

Bernardi, D. C., Mársico, E. T. de F., \& Queiroz, \&. M. (July/Aug 2013). El Método del Índice de Calidad para evaluar la frescura y la vida útil del pescado. SENASA. http://www.senasa.gob.ar/sites/default/files/ARBOL _SENASA/ANIMAL/ANIMALES\%20ACUATICOS/ INFO/BIBLIO\%20DIG\%20PESCA/Metodo_calidad_ evaluar_frescura_vida_util_pescado.pdf
Cheng, J.-H., Dai, Q., Sun, D.-W., Zeng, X.-A., Liu, D., \& \& $\mathrm{Pu}$, H.-B. (2013). Applications of non-destructive spectroscopic techniques for fish quality and safety evaluation and inspection. Trends in Food Science $\mathcal{E}$ Technology, 34(1), 18-31.

Cheng, J.-H., Sun, D.-W., Han, Z., \& Zeng, X.-A. (2014). Texture and Structure Measurements and Analyses for Evaluation of Fish and Fillet Freshness Quality: A Review. Comprehensive Reviews in Food Science and Food Safety, 13(1), 52-61.

Cheng, J.-H., Sun, D.-W., Zeng, X.-A., \& Liu, D. (2015). Recent advances in methods and techniques for freshness quality determination and evaluation of fish and fish fillets: a review. Critical Reviews in Food Science and Nutrition, 55(7), 1012-1225.

Choi, J.-W., Jang, M.-K., Hong, C.-W., Lee, J.-W., Choi, J.H., Kim, K.-B.-W.-R., Xu, X., Ahn, D.-H., Lee, M.-K., \& Nam, T. J. (2020). Novel application of an optical inspection system to determine the freshness of (mackerel) stored at a low temperature. Food Science and Biotechnology, 29(1), 103-107.

Chow, C.-F., Kong, H.-K., Leung, S.-W., Chiu, B. K. W., Koo, C.-K., Lei, E. N. Y., Lam, M. H. W., Wong, W.T., \& Wong, W.-Y. (2011). Heterobimetallic Ru(II)$\mathrm{Eu}(\mathrm{III})$ complex as chemodosimeter for selective biogenic amine odorants detection in fish sample. Analytical Chemistry, 83(1), 289-296.

Cropotova, J., Mozuraityte, R., Standal, I. B., Aftret, K. C., \& Rustad, T. (2019). The Effect of Sous-Vide Cooking Parameters, Chilled Storage and Antioxidants on Quality Characteristics of Atlantic Mackerel () in Relation to Structural Changes in Proteins. Food Technology and Biotechnology, 57(2), 191-199.

Cropotova, J., Mozuraityte, R., Standal, I. B., \& Rustad, T. (2019). Assessment of lipid oxidation in Atlantic mackerel (Scomber scombrus) subjected to different antioxidant and sous-vide cooking treatments by conventional and fluorescence microscopy methods. Food Control, 104, 1-8.

Cruces, C., Chero, J., Iannacone, J., Diestro, A., Sáez, G., \& \& Alvariño, L. (2014, July). Metazoans parasites of "chub mackerel" scomber japonicus houttuyn, 1782 (perciformes: scombridae) at the port of chicama, La Libertad, Perú. Neotropical Helminthology, 8(19951043). https://biblat.unam.mx/es/revista/neotropicalhelminthology/articulo/metazoos-parasitos-decaballa-scomber-japonicus-houttuyn-1782perciformes-scombridae-del-puerto-de-chicama-lalibertad-peru

De Alba, M., Bravo, D., \& Medina, M. (2012). High pressure treatments on the inactivation of Salmonella Enteritidis and the characteristics of beef carpaccio. Meat Science, 92(4), 823-828. 
ElMasry, G., \& Wold, J. P. (2008). High-speed assessment of fat and water content distribution in fish fillets using online imaging spectroscopy. Journal of Agricultural and Food Chemistry, 56(17), 7672-7677.

FAO. (1998). El pescado fresco: su calidad y cambios de su calidad. No. 348, Documento Técnico de Pesca, Roma. http://www.fao.org/3/v7180s/v7180s09.htm

FAO. (2014, May 19). El estado mundial de la pesca y la acuicultura 2014. Organización de Las Naciones Unidas Para La Alimentación Y La Agricultura. http://www.fao.org/resources/infographics/infograp hics-details/es/c/232019/

Fidalgo, L. G., Saraiva, J. A., Aubourg, S. P., Vázquez, M., \& \& Torres, J. A. (2014). Effect of high-pressure pretreatments on enzymatic activities of Atlantic mackerel (Scomber scombrus) during frozen storage. Innovative Food Science $\mathcal{E}$ Emerging Technologies: IFSET: The Official Scientific Journal of the European Federation of Food Science and Technology, 23, 18-24.

Gudjónsdóttir, M., Romotowska, P. E., Karlsdóttir, M. G., \& Arason, S. (2019). Low field nuclear magnetic resonance and multivariate analysis for prediction of physicochemical characteristics of Atlantic mackerel as affected by season of catch, freezing method, and frozen storage duration. Food Research International , $116,471-482$.

Hashimoto, K., Kobayashi, S., \& Yamashita, M. (2016). Comparison of connective tissue structure and muscle toughness of spotted mackerel Scomber australasicus and Pacific mackerel S. japonicus during chilled and frozen storage. Fish Sci, 83(133139). https://doi.org/10.1007/s12562-016-1042-4

Hashimoto, K., \& \& Yamashita, M. (2019). Seasonal variation in quality and chemical composition of the muscles of the spotted mackerel Scomber australasicus and Pacifc mackerel S. japonicus. Fisheries Science. https://doi.org/10.1007/s12562-01901324-0

Hernández-Martínez, M., Gallardo-Velázquez, T., OsorioRevilla, G., \& N Almaraz-Abarca \&. (2014). Application of MIR-FTIR spectroscopy and chemometrics to the rapid prediction of fish fillet quality. CyTA - Journal of Food, 12(4). https://doi.org/10.1080/19476337.2014.889213

He, S., Chen, Y., Yang, X., Gao, J., Su, D., Deng, J., \& Tian, B. (2020). Determination of biogenic amines in Chub Mackerel from different storage methods. Journal of Food Science, 85(6), 1699-1706.

IMARPE. (2019, December 23). Desarrollo de la pesquería de caballa (Scomber japonicus peruanus) durante el 2019, situación actual y perspectivas de explotación para el $2020 . \quad$ IMARPE. https://cdn.www.gob.pe/uploads/document/file/121 2722/Informe-correspondiente-Oficio-1071-2019IMARPE-DEC20200807-1746888-1sh4e5w.pdf

J. Ezquerra-Brauer, J. Miranda, A. Cepeda, J. Barros \& S. Aubourg. (2016). Effect of jumbo squid (Dosidicus gigas) skin extract on the microbial activity in chilled mackerel (Scomber scombrus). LWT - Food Science and Technology, 72, 134-140.

Kitchenham, B. (9 July, 2007). Guidelines for performing systematic literature reviews in software engineering. Technical Report. https://citeseerx.ist.psu.edu/viewdoc/download?doi $=10 \cdot 1 \cdot 1 \cdot 117.471 \&$ rep=rep1\&type $=$ pdf

Kunjulakshmi, S., Harikrishnan, S., Murali, S., D'Silva, J. M., Binsi, P. K., Murugadas, V., \& Samuel, M. P. (2020). Development of portable, non-destructive freshness indicative sensor for Indian Mackerel (Rastrelliger kanagurta) stored under ice. Journal of Food Engineering, 287, 110132.

Linares-Espinós, E., Hernández, V., Domínguez-Escrig, J. L., Fernández-Pello, S., Hevia, V., Mayor, J., PadillaFernández, B., \& Ribal, M. J. (2018). Methodology of a systematic review. Actas Urologicas Espanolas, 42(8), 499-506.

L. Otero, M. Pérez, F. Holgado, G. Márquez, \& E. López. (2019). Hyperbaric cold storage: Pressure as an effective tool for extending the shelf-life of refrigerated mackerel (Scomber scombrus, L.). Innovative Food Science $\mathcal{E}$ Emerging Technologies: IFSET: The Official Scientific Journal of the European Federation of Food Science and Technology, 51, 41-50.

Lv, R., Huang, X., Dai, C., Ye, W., \& \& Tian, X. (2019). A rapid colorimetric sensing unit for histamine content of mackerel using azo reagent. Journal of Food Process Engineering. https://doi.org/10.1111/jfpe.13099

Ministerio de Agricultura, A. y. M. A. (2017). El mercado de la caballa en España. Ministerio de Agricultura, Alimentación $\quad \mathrm{Y}$ Medio Ambiente. https://www.mapa.gob.es/es/pesca/temas/mercados -economia-pesquera/informecaballa2017_tcm30437224.pdf

Miramontes, J., Muñoz, M., Calvo-Manzano, J. A., \& Corona, B. (2016). Establecimiento del estado del arte sobre el aligeramiento de procesos de software. RISTI - Revista Ibérica de Sistemas E Tecnologias de Informação, 17, 16-25.

Moriya, K., Nakazawa, N., Osako, K., \& Okazaki, E. (2019). Effect of subzero temperature treatment at $-2{ }^{\circ} \mathrm{C}$ before thawing on prevention of thaw rigor, biochemical changes and rate of ATP consumption in frozen chub mackerel (Scomber japonicus). LWT, 114, 108396. 
Mozuraityte, R., Standal, I. B., Cropotova, J., Budźko, E., \& \& Rustad, T. (2020). Superchilled, chilled and frozen storage of Atlantic mackerel (Scomber scombrus) effect on lipids and low molecular weight metabolites. International Journal of Food Science $\mathcal{E}$ Technology. https://doi.org/10.1111/ijfs.14821

Muñoz, M., Peralta, M., \& Laporte, C. Y. (2019). Análisis de las debilidades que presentan las Entidades Muy Pequeñas al implementar el estándar ISO/IEC 29110: Una comparativa entre estado del arte y el estado de la práctica. RISTI - Revista Ibérica de Sistemas E Tecnologias de Informação, 34, 85-96.

Pazos, M., Méndez, L., Fidalgo, L. et al. (2015). Effect of High-Pressure Processing of Atlantic Mackerel (Scomber scombrus) on Biochemical Changes During Commercial Frozen Storage. Food Bioprocess Technol, 8. https://doi.org/10.1007/s11947-015-1567-z

Ponce-Corona, E., Sánchez, M. G., Fajardo-Delgado, D., Acevedo-Juárez, B., De-la-Torre, M., Avila-George, H., \& Castro, W. (2020). Una revisión sistemática de la literatura enfocada al uso de vehículos aéreos no tripulados durante el proceso de detección de vegetación. RISTI - Revista Ibérica de Sistemas E Tecnologias de Informação, 36, 82-101.

PRODUCE. (2020, October 29). Produce: Desembarque del Sector Pesca creció $81.4 \%$ en setiembre. PRODUCE. https://www.gob.pe/institucion/produce/noticias/31 1672-produce-desembarque-del-sector-pesca-crecio81-4-en-setiembre

Sarkardei, S., \& Howell, N. K. (2007). The effects of freezedrying and storage on the FT-Raman spectra of Atlantic mackerel (Scomber scombrus) and horse mackerel (Trachurus trachurus). Food Chemistry, 103(1), 62-70.

S. Aubourg, J. Torres, J. Saraiva, E. Guerra \& M. Vázquez. (2013). Effect of high-pressure treatments applied before freezing and frozen storage on the functional and sensory properties of Atlantic mackerel (Scomber scombrus). LWT - Food Science and Technology, 53(1), 100-106.

Senturk, T., \& \& Alpas, H. (2012). Effect of High Hydrostatic Pressure Treatment (HHPT) on Quality and Shelf Life of Atlantic Mackerel (Scomber scombrus). Food Bioprocess Technol, 6. https://doi.org/10.1007/s11947-012-0943-1

SIICEX. (2019). Ficha Comercial de la Caballa Congelada. SIICEX.
https://www.siicex.gob.pe/siicex/portal5ES.asp?_pa ge_=172.17100\&_portletid_=sfichaproductoinit\&scri ptdo $=$ cC_fp_init\&pproducto $=46 \&$ pnomproducto $=\mathrm{Ca}$ balla\%20congelada

Simeonidou, S., Govaris, A., \& Vareltzis, K. (1997). Quality assessment of seven Mediterranean fish species during storage on ice. Food Research International , 30(7), 479-484.

Sone, I., Skåra, S., \& \& Olsen, H. (2019). Factors influencing post-mortem quality, safety and storage stability of mackerel species: a review. European Food Research and Technology, 245. https://doi.org/10.1007/s00217018-3222-1

Stamatis, N., \& \& Arkoudelos, J. (2007). Quality assessment of Scomber colias japonicus under modified atmosphere and vacuum packaging. Food Control, 18(4), 292-300.

Suh, S., Kim, Y. E., Shin, D., \& Ko, S. (2017). Effect of frozen-storage period on quality of American sirloin and mackerel (Scomber japonicus). Food Sci Biotechnol, 26(1077-1084 ). https://doi.org/10.1007/s10068-017-0146-7

Sveinsdóttir, H. I., Karlsdóttir, M. G., Arason, S., Stefánsson, G., Sone, I., Skåra, T., Rustad, T., Larsson, K., Undeland, I., \& Gudjónsdóttir, M. (2020). Effect of antioxidants on the sensory quality and physicochemical stability of Atlantic mackerel (Scomber scombrus) fillets during frozen storage. Food Chemistry, 321, 126744.

Vázquez, M., Torres, J. A., Gallardo, J. M., Saraiva, J., \& Aubourg, S. P. (2013). Lipid hydrolysis and oxidation development in frozen mackerel (Scomber scombrus): Effect of a high hydrostatic pressure pretreatment. Innovative Food Science \& Emerging Technologies: IFSET: The Official Scientific Journal of the European Federation of Food Science and Technology, 18, 24-30.

Wu, L., Pu, H., \& \& Sun, D.-W. (2019). Novel techniques for evaluating freshness quality attributes of fish: A review of recent developments. Trends in Food Science E Technology, 83, 259-273.

Yu, D., Wu, L., Regenstein, J. M., Jiang, Q., Yang, F., Xu, Y., $\&$ Xia, W. (2020). Recent advances in quality retention of non-frozen fish and fishery products: A review. Critical Reviews in Food Science and Nutrition, 60(10), 1747-1759 